\title{
Diagnosing Salem University Lokoja Network for Better Network Performance
}

\author{
Onwudebelu Ugochukwu ${ }^{1, *}$, Datukun Kalamba Aristarkus ${ }^{2}$, S. E. Adewumi ${ }^{3}$ \\ ${ }^{1}$ Federal University Ndufu-Alike Ikwo (FUNAI) P.M.B. 1010, Abakaliki, Ebony State, Nigeria \\ ${ }^{2}$ ICT Unit, College of ICT Salem University (SU), Km 16, Lokoja-Ajaokuta Road, PMP 1060, Lokoja, Kogi State, Nigeria \\ ${ }^{3}$ Federal University Lokoja, (FUL) PMB 1154, Kogi State, Nigeria \\ *Corresponding Author: anelectuugocy@yahoo.com
}

Copyright (C) 2014 Horizon Research Publishing All rights reserved.

\begin{abstract}
With the increasing levels of deployment of various forms of high-speed (or broadband) services within today's Internet, there is new impetus to find some usable answers that allow both providers and users to place some objective benchmarks against the service offerings. Furthermore, with the lift in access speed with broadband services, there is an associated expectation on the part of the end user or service customer about the performance of the Internet service. It should be "better" in some fashion, where "better" relates to the performance of the network and the service profile that is offered to network applications. And not only is there an expectation of "better" performance, it should be measurable. This paper is concerned with the measurements of the network (IP Network) performance in Salem University and its necessary measurement results.
\end{abstract}

Keywords Network, Performance, Internet, Measurement, Traffic

\section{Introduction}

Before the advent of ICTs, University Libraries were using manual service systems for a variety of reasons such as adequacy in storage, access and retrieval of massive volume of information [14]. The provision of internet access is a basic need in any University environment due to the fact that teaching, research, administration and community services are more effectively carried out when there is a link to the internet [11]. As for digital technology involves the use of internet to collect, store, search and disseminate cultural, historical, economic and scientific information in a digitized form [1]. Information dissemination on the internet network is generally a silky venture due to the unsecure nature of the network [19]. Access to instruction through the Internet is flexible, ensures broad viability and availability of educational opportunities [2]. The benefits of Internet connectivity are quick and convenient information exchange, access to regular updates on topics of interest, access to experienced and expert individuals in thousands of fields [7].

With rapid growth of the Internet, the amount of traffic (real-time and non real- time) such as Voice over IP (VoIP) calls, multimedia and even non real-time applications like file transfer protocol (FTP), e-mails, etc have been increased. The requirements of real-time traffic in the Internet are; low loss rate and low end-to-end delay. Active queue management (AQM) such as tail drop (DT), Random early detection (RED) are usually used to control congestion in a router and quality of service (Qos) in the entire network [10]. Most of the time, the problem of network breakdown and "week network connection" in our communication systems are not only the problem of hardware components involved, but that of lack of cooperation among the remote objects that runs on different platforms and protocols [9]. Most internet users use electronic mail to communicate online. They depend on the email system to deliver their mails to the recipients [15]. Electronic mail undoubtedly doubles as one of the internet's most popular as well as killer application. A sizable percentage of emails circulating on the internet are unsolicited bulk emails called spam [8]. This has made some users unhappy, since most people depend on computer networks for their day-to-day activities to perform routine jobs, meaning that network must be available and offer good performance always [5].

Technologies for accurate bandwidth estimation are also important for traffic engineering, network troubleshooting and capacity planning support [18]. Internet penetration to Africa is less than $1 \%$ despite $12 \%$ world population. More than 400 ISPs have been licensed as well as a number of data carriers, interest exchange and gateway operators [6]. In the digital age, "connectivity" takes on broader meaning than simply putting two or more people in touch. The internet creates a new universal space for information sharing, collaboration and commerce [13]. Many companies started out small using homemade software and server facilities in their garages. Users of this service get a dial-up connection with an average speed of $9.6 \mathrm{kbps}$ to $14.4 \mathrm{kbps}$ and these connections were often not reliable. Accurate measurement of network bandwidth is important for network management 
applications as well as flexible internet applications and protocols which actively manage and dynamically adapt to changing utilization of network resources. Transport performances overlay network routing, peer-to-peer file distribution and verification of Service Level Agreements (SLA) [4]. The number of Internet users is difficult to gauge, but the number of hosts increased rapidly in recent years. From a little over 200 in 1981, the number of hosts increased to more than 300,000 by 1990 and reached 147 million in January, 2002; growing at a compound annual rate of 90 percent over 21 years [16].

Looking at the broad spectrum of performance, the answer is that speed is not everything. The ability of a network to support transactions that include the transfer of large volumes of data, as well as supporting a large number of simultaneous transactions, is also part of the overall picture of network load and hence of network performance. But large data set is not everything in performance. Consideration should also be given to the class of network applications where the data is implicitly clocked according to some external clock source. A functional description of network performance encompasses a description of speed, capacity, and distortion of transactions that are carried across the network. This informal description of what constitutes network performance certainly feels to be on the correct path, given that if one knows the latency, available bandwidth, and packet loss, as a profile of network performance between two network end points, as well as the characteristics of the network transactions, then it is possible to make a reasonable prediction relating to the performance of the transaction.

\section{Literature Review}

USA was responsible for developing networks through which the rest of the world was connected to form a global network of systems and the network was called the Internet. 1990 's Global Internetworking with the growth and development of the Internet became the need for speed - and bandwidth. A designed local area network (LAN) can equally provide user access to the Internet [17]. If a host is enabled with two network interfaces connected to the internet via two different Internet Service Providers (ISPs). Failures of one network will not stop data transmission. The host will still be capable of continuing the data transfer by switching over to the other network. If both networks are active at the same time, both packets experience high delay on one path [3]. In IP networks the ubiquitous network management tool is the Simple Network Management Protocol (SNMP) [12]. There is no doubt that SNMP can provide a wealth of data about the operational status of each management network element, but can it tell you anything about the overall network performance?

The most basic tool for measuring network performance is the periodic measurement of the interface byte counters. Such measurements can provide a picture of the current traffic levels on the network link, and when related to the total capacity of the link, the relative link loading level can be provided. As a performance indicator this relative link loading level can provide some indication of link performance, in that a relatively lightly loaded link (such as a load of 5 to 10 percent of total available capacity) would normally indicate a link that has no significant performance implications, whereas a link operating at 100 percent of total available capacity would likely be experiencing high levels of packet drop, queuing delay, and potentially a high jitter level. In between these two extremes there are performance implications of increasing the load. It should be noted that the characteristics of the link have a bearing on the interpretation of the load levels, and a low-latency 10-Gbps link operating at 90-percent load will have very significantly lower levels of performance degradation than a 2-Mbps high-latency link under the same 90-percent load.

Relative traffic load on each link can be complemented by measurement of performance-related SNMP counters. A management system can poll each active network element to retrieve the number of packets dropped for each interface, and the number of packets successfully forwarded. From these two data items, the relative drop proportion of packets can be calculated on an element-by-element and potentially a link-by-link basis and a series of element measures can provide a per-path drop proportion by combining the individual packet-forwarding measurements for the interfaces on the path. Because some count of relative packet drop rate can be gathered from each network element, with the additional input of the current forwarding state of the network it is possible to predict the path a packet will take through the network, and hence estimate the path probability of drop. However, this information is still well short of being a reliable measurement of service performance.

Another method for measuring network performance is queuing delay. Queuing delay is somewhat more challenging to measure on an element-by-element basis using element polling with SNMP. In theory, the polling system could use a rapid sequence of polling the output queue length of a router and estimating the queuing delay based on an average packet size estimate, together with the knowledge of the available output capacity. Of course, such a measurement methodology assumes a simple first-in, first-out (FIFO) queuing discipline, a queue size that varies slowly over time and slow link speeds. Such assumptions are rarely valid in today's IP networks. As the link speed increases, the queue size may oscillate with a relatively high frequency as a function of both the number and capacity of the input systems and of the capacity of the output system. In general, queuing delay is not easily measured using network element polling. There is no ready way for a polling mechanism to detect and count the incidence of reordered packets. Packet reordering occurs in many situations, including the use of parallel switching fabrics within a single network element and the use of parallel links between routers. IP routers are not typically designed to detect, let alone correct, packet reordering and because they do not detect this condition, they cannot report on the incidence of many packet reordering 
that occurs.

The complementary approach to performance instrumentation of network elements is active network probing. This requires the injection of marked packets into the data stream; collection of the packets at a later time; and correlation of the entry and exit packets to infer some information regarding delay, drop, and fragmentation conditions for the path traversed by the packet. The most common probe tools in the network today are ping and trace-route.

The best known and most widely used active measurement tool is ping. Ping is a very simple tool: a sender generates an Internet Control Message Protocol (ICMP) echo request packet, and directs it to a target system. As the packet is sent, the sender starts a timer. The target system simply reverses the ICMP headers and sends the packet back to the sender as an ICMP echo reply. When the packet arrives at the original sender's system, the timer is halted and the elapsed time is reported. A ping response indicates that the target host is connected to the network, is reachable from the query agent, and is in a sufficiently functional state to respond to the ping packet. In itself, this response is useful information, indicating that a functional network path to the target host exists. Failure to respond is not so informative because it cannot be reliably inferred that the target host is not available. The ping packet, or perhaps its response, may have been discarded within the network because of transient congestion, or the network may not have a path to the target host, or the network may not have a path back to the ping sending host, or there may be some form of firewall in the end-to-end path that blocks the ICMP packet from being delivered.

However, if you can ping a remote IP address, then you can obtain numerous performance metrics. Beyond simple reachability, further information can be inferred by the ping approach with some basic extensions to our simple ping model. If a sequence of labelled ping packets is generated, the elapsed time for a response to be received for each packet can be recorded, along with the count of dropped packets, duplicated packets, and packets that have been reordered by the network. Careful interpretation of the response times and their variance can provide an indication of the load being experienced on the network path between the query agent and the target. Load will manifest a condition of increased delay and increased variance, due to the interaction of the router buffers with the traffic flows along the path elements as load increases. When a router buffer overflows, the router is forced to discard packets; and under such conditions, increased ping loss is observed. In addition to indications of network load, high erratic delay and loss within a sequence of ping packets may be symptomatic of routing instability with the network path oscillating between many path states.

\section{Materials and Methods}

\subsection{Testing Methodology}

So far we have been looking at the ways of measuring network performance as a general task. Degraded performance does not happen by accident though sometimes accidents do happen, and it makes the measurement task easier if you can identify precisely what it is that you are looking for. This approach requires identification of the various situations that can impact network performance and then set up network measurement and monitoring systems that are tuned to identify these situations. Within this approach, the motives for network measurement are concerned with identification of traffic load patterns that cause uneven network load, monitoring, and verification of service-level agreements, detection of abnormal network load that may be a signature of an attack, forecasting and capacity planning, and routing stability [12].

Ping and trace-route are ubiquitous tools. Almost every device can support sending ping and trace-route probes, and, by default almost every device, including network routers, will respond to a ping or trace-route probe. One-way measurements are a different matter, and such measurements normally require the use of dedicated devices in order to undertake the clocking of the probes with the required level of precision. IP networks have busty traffic sources, and there is a marked self-similarity in the traffic patterns. This appears to be consistent over a wide range of networks, where large-capacity systems tend to observe large burst patterns and smaller systems also see bursts of a similar proportionate size. So the question is, what time interval for measurements can provide meaningful aggregation of information, while at the same time be sensitive enough to report on the outcomes of transient bursts within the network? Intuitively a measurement time base of hourly measurements is very insensitive to capturing transient bursts, whereas a time base of a millisecond would generate a massive amount of data, a scenario that would tend to smother the identification of abnormalities. Interestingly enough, the choice of a measurement base has little to do with the capacity of the links within a network, but it has a close relationship to the average routing trip time of the individual transport sessions that are active within the network.

Various tools were used to test or rather take a set of path measurements across the network. These include 'Ping' test, Ping Plotter and a Bandwidth Speed test tools. The Bandwidth of the University Network is 512MB upload/1meg down load.

\subsection{Network Utility - Ping}

Ping is a computer network administration utility used to test the reachability of a host on an Internet Protocol (IP) Network and to measure the RTT for messages sent from the original host to a destination computer. Thus, the gateway, the wireless router, yahoomail.com and google.com was 'pinged' in order to test the Internet and LAN connectivity for latency and attenuation as indicated in Figure 4. The Round Trip Time [20] (also known as the round trip delay time (RTD) or ping time or latency) is the length of time it 
takes for a signal to be sent plus the length of time it takes for an acknowledgement of that signal to be received. That is, it time delay consists of the transmission times between the two points of a signal. This is determined by using the ping command. While Time to Live (also known as hop limit is a mechanism that lime the lifespan or life time of data in a computer or network, this help in preventing a data packet from circulating indefinitely) [21].

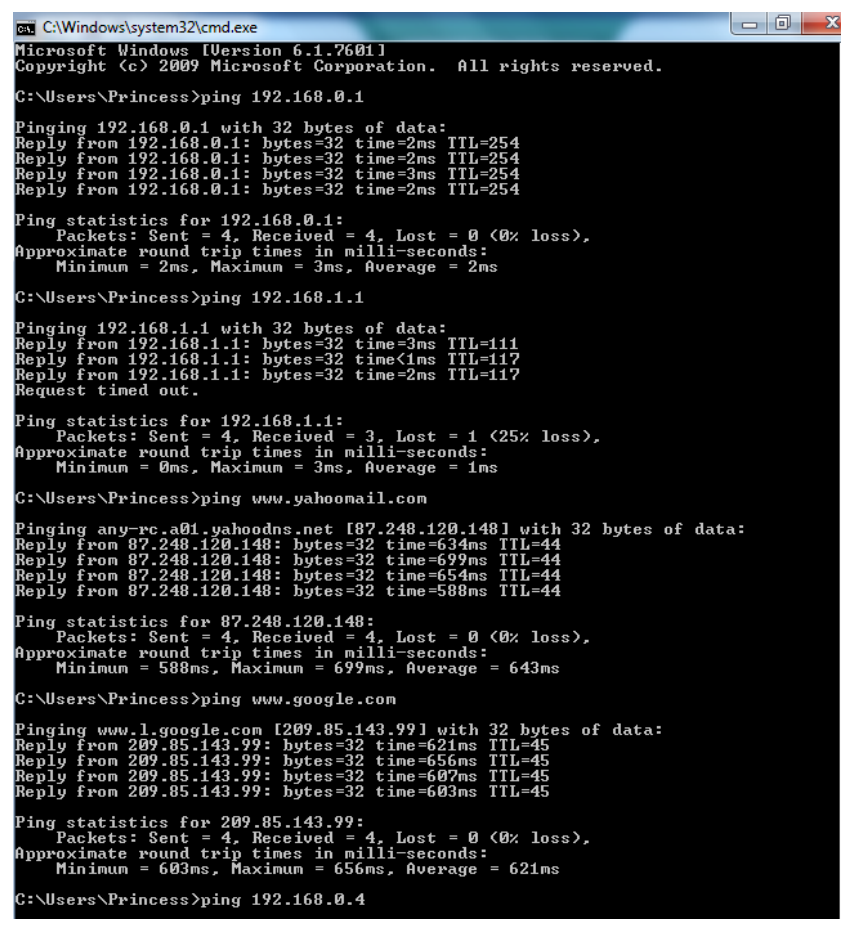

Figure 1. Outputs of 'Ping' Connectivity Test

The information in Figure 1 above can be represented in a tabular form for more clarity as shown below. The performance metrics of interest to us are: Time-To-Live and Round-Trip-Time indicated which have been illustrated in the tables 1-5 and also shown in Figures 2-5.

Table 1. Gateway (192.168.0.1) 'Ping' Test Output

\begin{tabular}{|c|c|}
\hline R T T (Milliseconds) & TTL \\
\hline 2 & 254 \\
\hline 2 & 254 \\
\hline 3 & 254 \\
\hline 2 & 254 \\
\hline
\end{tabular}

Table 2. A network interface (192.168.1.1) 'Ping' Test Output

\begin{tabular}{|c|c|}
\hline RTT (Milliseconds) & TTL \\
\hline 3 & 111 \\
\hline 1 & 117 \\
\hline 2 & 117 \\
\hline 0 & 0 \\
\hline
\end{tabular}

Table 3. www.yahoomail.com 'Ping' Test Output

\begin{tabular}{|c|c|}
\hline RTT (Milliseconds) & TTL \\
\hline 634 & 44 \\
\hline 699 & 44 \\
\hline 654 & 44 \\
\hline 588 & 44 \\
\hline
\end{tabular}

Table 4. www.google.com 'Ping' Test Output

\begin{tabular}{|c|c|}
\hline RTT (Milliseconds) & TTL \\
\hline 621 & 45 \\
\hline 656 & 45 \\
\hline 687 & 45 \\
\hline 683 & 45 \\
\hline
\end{tabular}

The charts for table 1 is shown in Figure 2, that of table 2 in Figure 3, that of table 3 in Figure 4 while that of table 4 in Figure 5 below, which were plotted using Microsoft Excel. Series 1 represents the RTT while Series 2 is for TTL as shown in the charts.

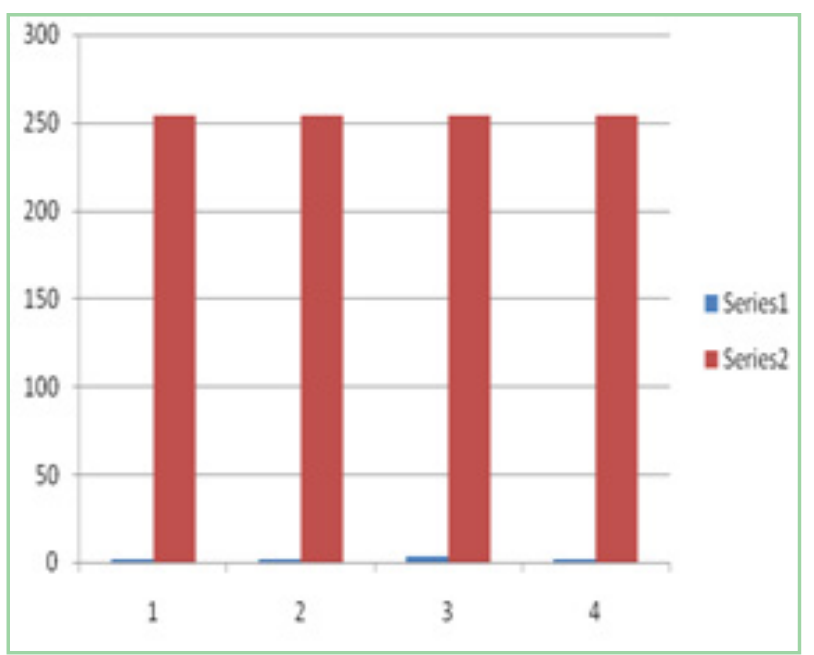

Figure 2. Round-Trip-Time and Time-to-live chart from table 1

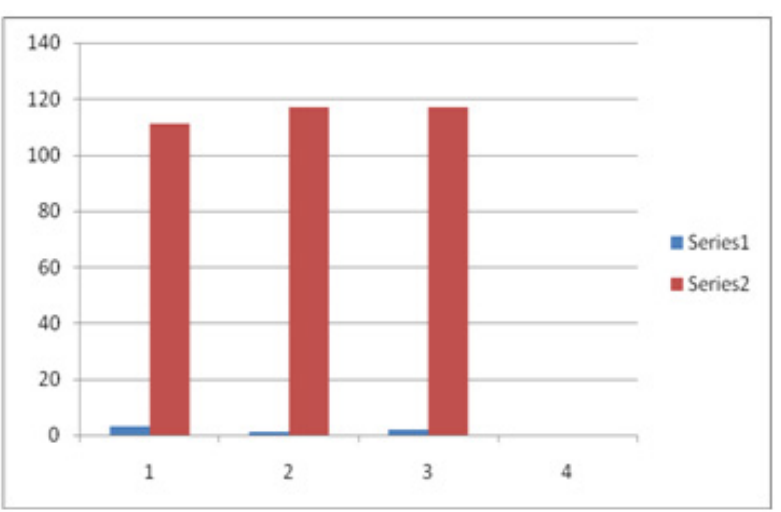

Figure 3. Round-Trip-Time and Time-to-live Chart from table 2 


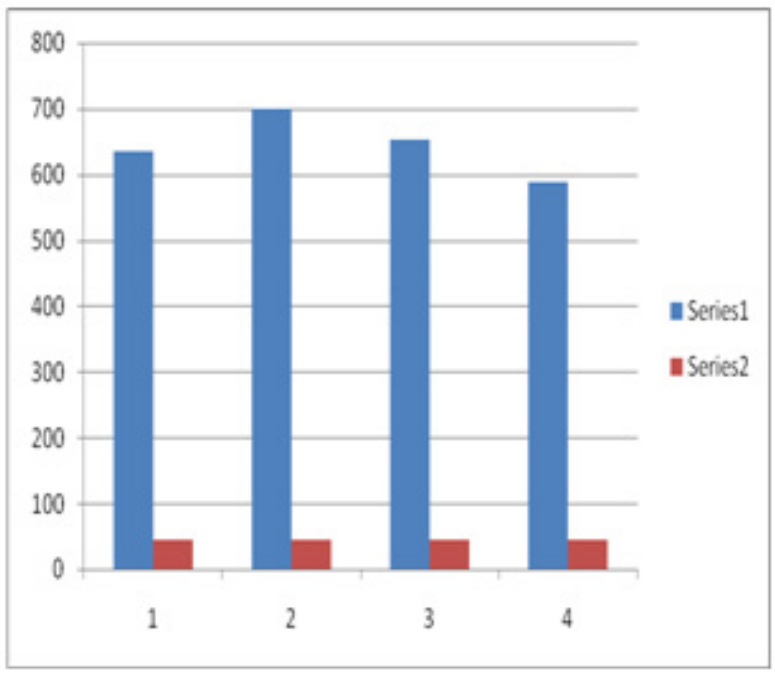

Figure 4. Round-Trip-Time and Time-to-live Chart from table 3

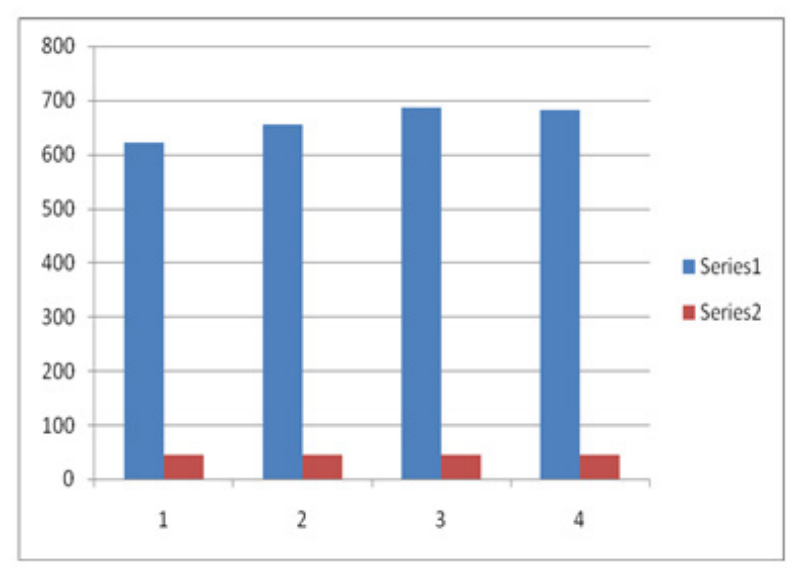

Figure 5. Round-Trip-Time and Time-to-live Chart from table 4

In the charts (Figures 2 to 5 ) above, series 1 refers to the RTT (latency) while series 2 refers to the TTL, used to avoid packets loops as mentioned above. Figures 2 and 3 have higher TTL than RTT whereas Figures 4 and 5 have higher RTT than TTL. The smaller the RTT score means a faster the connection. The higher the RTT the more delay (latency) in the time packets travel a full round trip , therefore, Figures 2 and 3 have less latency (delay) in transmission compare to Figures 4 and 5.

\subsection{Diagnostic Tool - Ping Plotter}

Ping Plotter is a network troubleshooting and diagnostic tool. It uses a combination of trace-route, ping and whois to collect data quickly and then allows you to continue to collect data over time. It helps to pinpoint where the problem in an internet connection is coming from, in an intuitive graphical way and to continue monitoring your connection long-term to further identify issues. As stated in [22] Ping Plotter offers some unique value for network monitoring and troubleshooting amongst which include graphically display performance metrics about the route your data takes to a server; monitor network performance over time, capturing the moments when problems surface; get notified when there is a network problem.

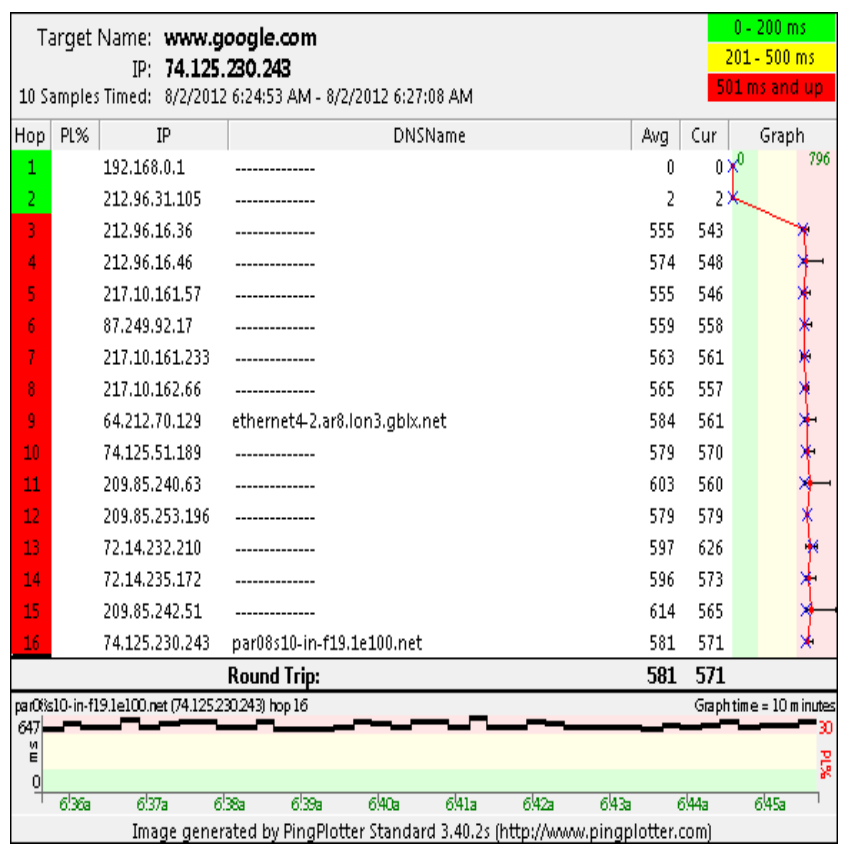

Figure 6. The output of tracing www.google.com on ping plotter standard

The ping plotter report above shows where the information is being sent (i.e. tracing www.google.com which is the target name) through the internet and what is happening along the way. The tool use to generate the above report is a lightweight network troubleshooting, diagnostic and monitoring tool called a PingPlotter standard. From Figure 6, there are upper and lower graph corresponding to trace and time graph respectively. On the trace graph, the thresholds set for the colors on the trace graph background (with $200 \mathrm{~ms}$ as warning color and $500 \mathrm{~ms}$ as critical speed which is ping plotter's defaults), the red line on the graph represents the average response time. The blue $\mathrm{x}$ represents the response time for the current packet, the black horizontal line represents the minimum and maximum response times, Avg. column is for average response time, Cur. (Current) column is for individual sample time for the most recent sample (ERR displayed in this column connotes lost packets). DNS column is for that hop (in this column means an unresolved name), IP column is the IP addresses of various hobs. PL\% column refers to packet lost by individual hobs, Hop column is for the individual hobs in serial. Graph is the trace graph itself.

In the case of the time graph (TG), the time it takes for a ping to get from my computer to the target device and back (Round Trip time) is 581 and 571 for Avg. and Cur respectively. Latency scale is $60 \mathrm{~ms}$ at the left maximum while the packet lost is $30 \mathrm{~ms}$ at the right maximum. The graph time is 10 minutes. A software call ping plotter is used to determine the trace and time graph described to determine the rate of delay in packets delivery. 


\subsection{Outputs of bandwidth Speed Test Carried out for 3 Days}

Bandwidth is the amount of data that one is allowed to download/upload at any given time. The bandwidth speed test is carried out to determine download and upload speeds for the university Internet connection, thus the internet speed. The unit for the bandwidth speed is kbps (Kilo Bits per Second) and it is the rate at which 1000 bits of data travels across an internet connection. (Remember a bit is the smallest unit of digital information, while 8 bits make up 1 byte). The test shows the current throughput you can get on ones internet connection. Factors such as network congestion between the University and the servers; line quality, distance from exchange, technology used, hardware capabilities, server route and other downloads in progress can affect the available throughput. The Bandwidth Speed Test was carried out using a bandwidth online speed meter or checker from www.bandwidthplace.com and www.ip-address.com/speedtest/ and the results are tabulated as follows:

Table 5. Bandwidth Speed Test Result for Day One

\begin{tabular}{|c|c|c|c|}
\hline Category & 1st Test (Kbps) & 2nd Test (Kbps) & 3rd Test (Kbps) \\
\hline Upload & 116 & 123 & 121 \\
\hline Download & 912 & 933 & 964 \\
\hline
\end{tabular}

Table 6. Bandwidth Speed Test Result for Day Two

\begin{tabular}{|c|c|c|c|}
\hline Category & 1st test (Kbps) & 2nd Test (Kbps) & 3rd Test (Kbps) \\
\hline Upload & 85 & 25 & 35 \\
\hline Download & 24 & 25 & 35 \\
\hline
\end{tabular}

Table 7. Bandwidth Speed Test Result for Day Three

\begin{tabular}{|c|c|c|c|}
\hline Category & 1st test (Kbps) & 2nd Test (Kbps) & 3rd Test (Kbps) \\
\hline Upload & 174 & 174 & 204 \\
\hline Download & 983 & 942 & 931 \\
\hline
\end{tabular}

Tables 5 and 7 have higher download speed than upload speed over table 6 . In table 6 , the upload has higher bandwidth speed; the entire bandwidth speed is slow compare to table 5 and 7.

The Charts below depicts the graphs of results of various tests carried out on the current Internet service bandwidth on Salem University campus. Series 1 refers to the download speed while Series 2 refers to the upload speed. Both of them are plotted against the number of times the test is being carried out. Microsoft excel was used to plot the graphs below. From table 5, the Figure 7 below is the bandwidth test at a time for day one. The test is carried out using bandwidth speed test software directly from online as stated above.

From table 6, Figure 8 below is the bandwidth test at a time for day two:

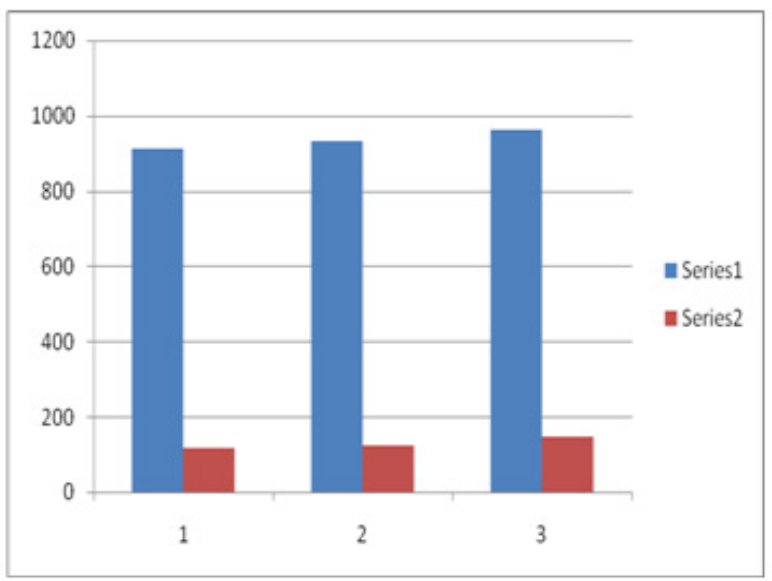

Figure 7. Chart of Bandwidth against Number of Times Test is carried out

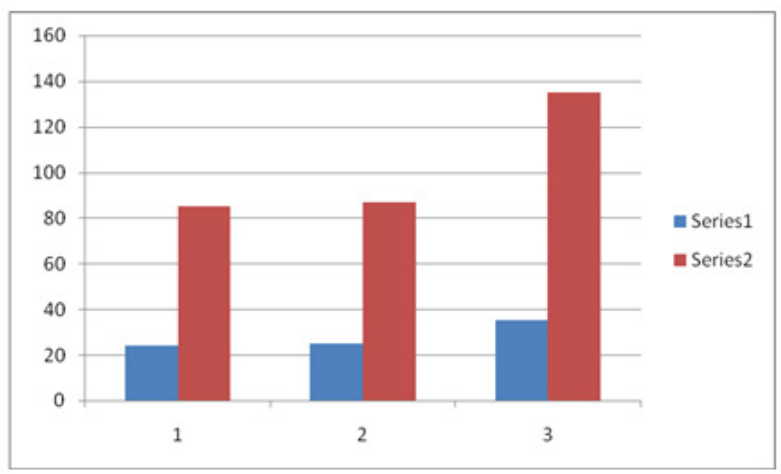

Figure 8. Chart of Bandwidth against Number of Times Test is Carried out

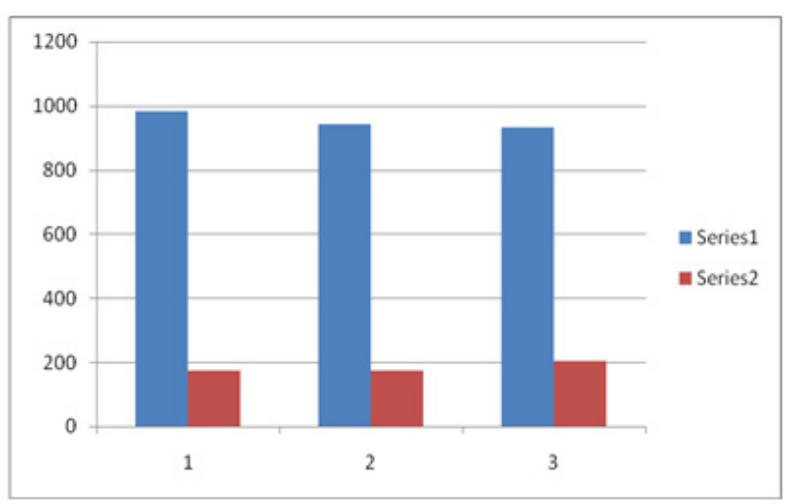

Figure 9. Chart of Bandwidth against Number of Times Test is carried out

From table 7, Figure 9 above is the bandwidth test at a time for day three.

\section{Conclusion}

Generally, we shall deduce that the round Trip Time (RTT) is average which virtually connotes average delay in signal transmission after the whole test exercise. The ping plotter output depicts higher RTT on the average, likewise "Ping Connectivity Test". "Bandwidth speed test" entails the rate 
of bandwidth in transmission on the Network. The higher the bandwidth the lower the delay (latency), hence more speed. Since the actual bandwidth in question is $512 / 1 \mathrm{meg}$, we only have good download speed compare to upload speed with reference to their original rate, hence average delay is ascertained. The higher the RTT the more delay (latency) at the time packets travel a full round trip, hence the instance where we have higher RTT on the test output, is on the average side. On the Campus under this research, higher bandwidth will have to be subscribed to in order to sustain lesser delay in signal transmission on the Campus' Network.

\section{Acknowledgements}

We are grateful to the management of Salem University Lokoja for helping us collect data from their ICT Unit. We also thank Moses Princess Ufouma for providing some of the tools used in the research work and helpful discussions which helped improve this paper.

\section{REFERENCES}

[1] O. A. Abisoye, Challenges facing the digitization of University Libraries in Nigeria; a case study of Federal University of Technology, Mina: An international Journal of Information and Communication Technology (ICT), The Pioneer Journal in Africa, Vol. 7(2), pp. 35-36, 2010.

[2] E.I. Adebayo, Information Technology for People-Centered Development: Potentials Realized Solution and use of E-Learning in Vocational Training; a way forward in amnesty programme in Niger-Delta Region of Nigeria Information Technology for People-Centred Development (ITePED), Nigeria Computer Society Conference Proceedings, Vol. 22, pp. 17-22, 2011.

[3] S. D. Adeniji, Routing Message For Mobility Management in Vehicle IPV6 Networks, International Conference on ICT for Africa 2011 (ICT4A 2011), Vol. 3, pp. 238 - 242, 2011.

[4] O. S. Adewale, O. S. Ogundele, and B. K. Alese, A Measurement System For Monitoring Clients Bandwidth Usage, International Conference on ICT for Africa 2011 (ICT4A 2011), Vol. 3, pp. 176-182, 2011.

[5] S. A. Akinboro, M. A., Adegoke, E. A. Olajubu, and A. O. Ajayi, Adaptive Filtering Approach for Fault Management in Bells Wireless Campus Area Network, International Conference on ICT for Africa 2011 (ICT4A 2011), Vol. 3, pp. 53-58, 2011.

[6] R. O. Akinyede, O. K. Boyinbode, and B. K. Alese, Poverty reduction in Nigeria using Information Technology, 9th International Conference on Managing Current Global Challenges: Information Technology Strategies and Tools (MacGlobe'09), Vol. 20, pp. 3-12, 2009.

[7] M. Alasa and K. Ibbenne, Internet and academic Library Services development in Nigeria; a paper presented at the National Conference and annual General meeting of the Nigerian Library Association, pp. 4-8, 1998.
[8] K.. Albercht, Mastering Spam, A multifaceted approach with the Spamato Spam Filter System DSS. ETH NO16839 out-law news, 2006

[9] F. O. Aranuwa, and A. Salami, Remote Function Calls And Interactions Among Remote Objects In Distributed Communication, Systems, International Conference on ICT for Africa 2011 (ICT4A 2011), Vol. 3, pp. 29-34, 2011.

[10] S. Chaudhuri, Performance Enhancement in REM using Adaptive Drop Policy for Protective and Best Effort, Springer Verlag, Berlin Heidetberge, pp. 3385-390, 2006.

[11] O. Dele, D. K. Aristarkus, and M. K. Aregbesola, Technical Factors Relevant to Internet Service Provision in a Typical Nigerian University, Information Technology for People-Centred Development (ITePED), Nigeria Computer Society Conference Proceedings, Vol. 22, pp. 23-29, 2011.

[12] H. T. Geoff, The Internet Protocol Journal, Vol. 6(1), 2003.

[13] A. B. Obotivere, G. O.Eloho, and F. Elugwu, Enabling Digital Technology for National Development, 9th International Conference on Managing Current Global Challenges: Information Technology Strategies and Tools (MacGlobe'09), Vol. 20, pp. 189-192, 2009.

[14] A. A. Oduwole, and C. B. Akpati, Accessibility and retrieval of electronic information at the University of Agriculture Library, Library review, Vol. 52(2), pp. 228-233, 2003.

[15] A. O. Okunade, A. B. C. Robert, O. B. Longe, and O. F. W. Onifade, Word-Statement Algorithm to Improve Bayesian Classification of Electronic Spam Mails, 9th International Conference on Managing Current Global Challenges: Information Technology Strategies and Tools (MacGlobe'09), Vol. 20, pp. 215-220, 2009.

[16] A. Omotosho, O. J. Emuoyibofarhe, and O. Adegboola, ICT in Health Care System; a Framework for Developing Nations, International Conference on ICT for Africa 2011 (ICT4A 2011), Vol. 3, pp. 95-100, 2011.

[17] O. J. Oyelade, Internet Concepts and Web Design, National Open University of Nigeria, pp. 13-19, 2010.

[18] R.S. Prasad, Bandwidth estimation, metrics, measurement, techniques and tools. IEEE networks, 2003.

[19] O. W. Victor, Information Technology for People-Centered Development: Information Dissemination on the internet in the Nigerian based on some functions of cryptography, Information Technology for People-Centred Development (ITePED), Nigeria Computer Society Conference Proceedings, Vol. 22, pp. 3-8, 2011.

[20] www.en.wikipedia.org/wiki/round_trip_time

[21] www.en.wikipedia.org/wiki/time to live

[22] www.pingplotter.com 